\title{
Speed of reaction-diffusion fronts in spatially heterogeneous media
}

\author{
Vicenç Méndez, ${ }^{1}$ Joaquim Fort, ${ }^{2}$ Horacio G. Rotstein, ${ }^{3}$ and Sergei Fedotov ${ }^{4}$ \\ ${ }^{1}$ Departament de Medicina, Facultat de Ciències de la Salut, Universitat Internacional de Catalunya, c/ Gomera s/n, \\ 08190-Sant Cugat del Vallès (Barcelona), Spain \\ ${ }^{2}$ Departament de Física, Universitat de Girona, Campus Montilivi, 17071 Girona, Catalonia, Spain \\ ${ }^{3}$ Department of Mathematics and Center for Biodynamics, Boston University, Boston, Massachusetts 02215, USA \\ ${ }^{4}$ Department of Mathematics, UMIST-University of Manchester, Institute of Science and Technology, \\ Manchester M60 1DQ, United Kingdom
}

(Received 16 September 2002; revised manuscript received 15 May 2003; published 13 October 2003)

\begin{abstract}
The front speed problem for nonuniform reaction rate and diffusion coefficient is studied by using singular perturbation analysis, the geometric approach of Hamilton-Jacobi dynamics, and the local speed approach. Exact and perturbed expressions for the front speed are obtained in the limit of large times. For linear and fractal heterogeneities, the analytic results have been compared with numerical results exhibiting a good agreement. Finally we reach a general expression for the speed of the front in the case of smooth and weak heterogeneities.
\end{abstract}

DOI: 10.1103/PhysRevE.68.041105 PACS number(s): 82.20.Fd, 05.40.Jc, 82.40. - g, 05.60.Cd

\section{INTRODUCTION}

Front propagation modeled by reaction-diffusion equations has been applied in many areas of science such as physics, biology, ecology, and chemistry [1,2]. Since the pioneering works by Kolmogorov, Petrovskii, and Piskunov (KPP) [3] and Fisher [4], both in 1937, this field has been continuously growing. The basic phenomena have been described by using parabolic reaction-diffusion equations derived under the assumption that the medium in which fronts are moving is homogeneous. Although heterogeneities are always present in nature, studies of fronts in heterogeneous media have been much more recent. Some examples are porous media, random media, noisy media, ecological patchiness, etc.

Experimental studies have been developed for heterogeneous excitable media. In this context, two-dimensional front propagation in the photosensitive Belousov-Zhabotinsky modulated reaction $[5,6]$ and patchy media [7] have been explored. Successful theoretical efforts have been also made to understand the phenomenon of front propagation in excitable media. In particular, Xin [8] has studied front solutions for reaction-diffusion equations in periodic and random media [8], and Shigesada et al. [9] have given analytical restrictions for the existence of propagating fronts in ecological patchy environments. Heterogeneous models have been also used, via computer simulations, to describe the dynamics of brain tumors [10]. Moreover, diffusion coefficients depending on spatial and temporal coordinates have been recently proposed to study the formation of Alzheimer's disease senile plaques [11]. Nakamura et al. [12], have studied reaction-diffusion equations when the spatially inhomogeneous reaction rate is much larger than the diffusion coefficient. Keener [13,14], Mitkov et al. [15] and Rotstein et al. [16] have studied bistable-type models in heterogeneous media in the context of calcium release waves [17]. Petrovskii has analyzed the case of a spatially periodic environment [18]. Many authors have recently studied the effect of the external multiplicative noise on the speed and the width of the front. These studies may be seen as a way to introduce stochastic heterogeneities in the reaction-diffusion equation through a control parameter [19]. Therefore, the effect of heterogeneities on front propagation is of wide theoretical and practical interest.

Our goal in this work is to understand how deterministic heterogeneities influence the front speed of parabolic reaction-diffusion equations with a monostable reaction term, when either the diffusion coefficient or the reaction term depend on the spatial coordinate. Methods such as marginal (linear) stability [20] and variational [21] analysis have been widely used to find the asymptotic speed of a front. However, both methods do not hold, or at least they should be adequately generalized, when the reaction-diffusion equation has an explicit dependence on the spatial coordinate. Instead, we will make use of well-known analytical techniques such as singular perturbation analysis and the local speed approach, both valid for weak heterogeneities, and geometrical methods for general heterogeneities, in order to study how heterogeneities introduce corrections to the asymptotic front speed, both for pulled (KPP) and pushed (but monostable) fronts. We will also compare the analytical results and numerical simulations.

The methods we employ here have some limitations. Singular perturbative analysis may be efficiently compared to numerical results when the solution to the leading order is known and for reaction-diffusion equations with non-KPP kinetic terms. The solution for the lowest order may be found for some particular non-KPP kinetic terms but it is not known in general, although in those cases numerical solution may always be calculated. This method requires, of course, a small parameter present in the model. Therefore, it is necessary to assume that the spatial heterogeneities of the media introduce a small variation in the reaction rate and/or the diffusion coefficient (weak heterogeneities) and the characteristic length of the heterogeneities must be greater than the characteristic width of the front (smooth heterogeneities) [22]. On the other hand, the geometrical method we present here, based on Hamilton-Jacobi dynamics, only holds for 
KPP kinetics but, contrary to the previous method, there is no need to assume either weak or smooth heterogeneities. The local speed approach is based in the assumption that for weak and smooth heterogeneities the speed of the front is given by the local value of the reaction rate $U$ and/or the diffusion coefficient $D$ in each spatial point, i.e., the front speed would be $v \simeq 2 \sqrt{U(x) D(x)}$ for KPP kinetics. Therefore, fronts with $U=b$ (constant) and $D=f(x)$ would have $v \simeq 2 \sqrt{b f(x)}$, and the same would hold for fronts in media with $U=f(x)$ and $D=b$. But, as we shall see, in general this simple approach is not consistent either with our analytical results or with our numerical simulations.

In this paper we study first the dynamics of front motion for the following smooth heterogeneous problems

$$
\begin{gathered}
\partial_{t} \phi=\partial_{x x} \phi+U(\varepsilon x) f(\phi), \\
\partial_{t} \phi=\partial_{x}\left[D(\varepsilon x) \partial_{x} \phi\right]+f(\phi),
\end{gathered}
$$

where the function $f$ satisfies $f(0)=f(1)=0, \quad \phi(x, 0)$ $=\theta(x)$ where $\theta(x)$ is an initial condition that may range from the Heaviside step function $[\phi(x, 0)=1$ for $x<0$ and $\phi(x, 0)=0$ for $x>0$ ] to a fully developed front, $D$ and $U$ are the dimensionless diffusion coefficient and reaction rate, respectively, and $\varepsilon$ is a small parameter. Since we expect solutions to behave like totally developed fronts, we should look at them in the asymptotic regime (large-space and largetime limit) $t \rightarrow t / \varepsilon$ and $x \rightarrow x / \varepsilon$. The scaling considered is equivalent to assuming that the front is totally developed independent of the way it developed from initial conditions. Equations (1) then become

$$
\begin{gathered}
\varepsilon \partial_{t} \phi=\varepsilon^{2} \partial_{x x} \phi+U(x) f(\phi), \\
\varepsilon \partial_{t} \phi=\varepsilon^{2} \partial_{x}\left[D(x) \partial_{x} \phi\right]+f(\phi) .
\end{gathered}
$$

Consistent with the initial conditions and the existence of a front we require the solution to satisfy $\lim _{x \rightarrow-\infty} \phi=1$ and $\lim _{x \rightarrow \infty} \phi=0$.

\section{NONUNIFORM REACTION}

We consider the problem

$$
\begin{gathered}
\varepsilon \partial_{t} \phi=\varepsilon^{2} \partial_{x x} \phi+U(x) f(\phi), \\
U(x) \equiv 1+\delta \eta_{u}(x)
\end{gathered}
$$

where $\delta$ is the amplitude of the heterogeneities and $\eta_{u}(x)$ is the reactive heterogeneity.

\section{A. Singular perturbation analysis}

This method of perturbative analysis has been already employed to study the speed of pulled fronts and it has been shown that the solvability integrals diverge [23]. Therefore, we will use this method only for non-KPP kinetics. We assume $\delta=O(\varepsilon)$ (weak heterogeneities), i.e., $\delta \equiv \sigma \varepsilon$, where $\sigma=O(1)$ in Eq. (3). Equation (3), together with the corresponding boundary conditions, becomes

$$
\begin{gathered}
\varepsilon \partial_{t} \phi=\varepsilon^{2} \partial_{x x} \phi+\left[1+\sigma \varepsilon \eta_{u}(x)\right] f(\phi), \\
\lim _{x \rightarrow-\infty} \phi=1, \quad \lim _{x \rightarrow \infty} \phi=0 .
\end{gathered}
$$

In order to study Eqs. (4) we will make a nonrigorous asymptotic analysis. We assume that the domain is divided into two regions according to the space scales: a boundary layer region, whose width is $O(\varepsilon)$, in which $\phi$ is rapidly varying; and an external region in which $\phi$ is almost constant, i.e., either $\phi=O\left(\varepsilon^{n_{1}}\right)$ or $\phi=1+O\left(\varepsilon^{n_{2}}\right)$, where $n_{1}$ and $n_{2}$ are positive real numbers.

In order to solve Eq. (4) in the outer region we expand $\phi$ as follows:

$$
\phi(x, t ; \varepsilon)=\Phi_{0}(x, t)+\varepsilon \Phi_{1}(x, t)+\varepsilon^{2} \Phi_{2}(x, t)+O\left(\varepsilon^{3}\right) .
$$

By substituting Eq. (5) into Eq. (4) and collecting terms with the same powers of $\varepsilon$ we get

$$
\begin{gathered}
f\left(\Phi_{0}\right)=0, \quad \lim _{x \rightarrow-\infty} \Phi_{0}=1, \quad \lim _{x \rightarrow \infty} \Phi_{0}=0, \\
\partial_{t} \Phi_{0}=f^{\prime}\left(\Phi_{0}\right) \Phi_{1}+\sigma \eta_{u}(x) f\left(\Phi_{0}\right), \quad \lim _{x \rightarrow \pm \infty} \Phi_{1}=0,
\end{gathered}
$$

and

$$
\begin{aligned}
\partial_{t} \Phi_{1}= & \partial_{x x} \Phi_{0}+\frac{1}{2} f^{\prime \prime}\left(\Phi_{0}\right) \Phi_{1}^{2}+f^{\prime}\left(\Phi_{0}\right) \Phi_{2} \\
& +\sigma \eta_{u}(x) f^{\prime}\left(\Phi_{0}\right) \Phi_{1}, \quad \lim _{x \rightarrow \pm \infty} \Phi_{2}=0 .
\end{aligned}
$$

The solution of Eq. (6) is $\Phi_{0}=1$ to the left of the boundary layer and $\Phi_{0}=0$ to the right of the boundary layer. The solutions of Eqs. (7) and (8) are $\Phi_{1} \equiv 0$ and $\Phi_{2} \equiv 0$, respectively. Thus, $\phi(x, t ; \varepsilon)=O\left(\varepsilon^{3}\right)$ to the left of the boundary layer and $\phi(x, t ; \varepsilon)=1+O\left(\varepsilon^{3}\right)$ to the right of the boundary layer. Note that to the order of magnitude considered here, there is no effective difference between the homogeneous and heterogeneous cases, i.e., there is no difference in the shape of the front.

In order to study the dynamics in the interior of the boundary layer we translate Eq. (4) to the reference frame of the front, i.e., we define the new variable $z=[x-S(t)] / \varepsilon$ where $S(t)$ represents the position of the front. The derivatives in Eqs. (2) transform according to

$$
\begin{gathered}
\partial_{t} \rightarrow-\frac{\dot{S}}{\varepsilon} \partial_{z}+\partial_{t}, \\
\partial_{x x} \rightarrow \frac{1}{\varepsilon^{2}} \partial_{z z},
\end{gathered}
$$

where the dot symbol stands for the temporal derivative. We expand $\phi$ and $S$ in powers of $\varepsilon$ : 


$$
\begin{gathered}
\phi(z, t)=\phi_{0}(z)+\varepsilon \phi_{1}(z, t)+\varepsilon^{2} \phi_{2}(z, t)+\cdots, \\
S(t)=S_{0}(t)+\varepsilon S_{1}(t)+\varepsilon^{2} S_{2}(t)+\cdots
\end{gathered}
$$

and, in consequence,

$$
\begin{aligned}
\eta_{u}(x)= & \eta_{u}\left(S_{0}+z \varepsilon+S_{1} \varepsilon+\cdots\right) \simeq \eta_{u}\left(S_{0}\right)+\eta_{u}^{\prime}\left(S_{0}\right)\left(z+S_{1}\right) \varepsilon \\
& +\cdots, \\
f(\phi)= & f\left(\phi_{0}\right)+f^{\prime}\left(\phi_{0}\right) \phi_{1} \varepsilon+\frac{1}{2} f^{\prime \prime}\left(\phi_{0}\right) \phi_{1}^{2} \varepsilon^{2}+f^{\prime}\left(\phi_{0}\right) \phi_{2} \varepsilon^{2} \\
& +\cdots,
\end{aligned}
$$

where $\quad \eta_{u}^{\prime}\left(S_{0}\right)=d \eta_{u}(x) /\left.d x\right|_{x=S_{0}} \quad$ and $\quad f^{\prime}\left(\phi_{0}\right)=d f(\phi) /$ $\left.d \phi\right|_{\phi=\phi_{0}}$. Inserting Eqs. (10) and (11) into Eq. (4) once Eqs. (9) are taken into account, and collecting terms with equal powers of $\varepsilon$ one gets $O(1), O(\varepsilon)$, and $O\left(\varepsilon^{2}\right)$, respectively:

$$
\begin{gathered}
\mathcal{L}\left(\phi_{0}\right)=0, \\
\mathcal{L}_{1}\left(\phi_{1}\right)=-\sigma f\left(\phi_{0}\right) \eta_{u}\left(S_{0}\right)-\dot{S}_{1} \partial_{z} \phi_{0}, \\
\mathcal{L}_{1}\left(\phi_{2}\right)=-\dot{S}_{2} \partial_{z} \phi_{0}-\dot{S}_{1} \partial_{z} \phi_{1}-\frac{1}{2} f^{\prime \prime}\left(\phi_{0}\right) \phi_{1}^{2} \\
-\sigma \eta_{u}\left(S_{0}\right) f^{\prime}\left(\phi_{0}\right) \phi_{1} \\
-\sigma f\left(\phi_{0}\right) \eta_{u}^{\prime}\left(S_{0}\right)\left(z+S_{1}\right)+\partial_{t} \phi_{1},
\end{gathered}
$$

where $\mathcal{L}=\partial_{z z}+\dot{S}_{0} \partial_{z}+f\left(\phi_{0}\right)$ and $\mathcal{L}_{1}=\partial_{z z}+\dot{S}_{0} \partial_{z}+f^{\prime}\left(\phi_{0}\right)$.

Since we assume $\phi_{0}=\phi_{0}(z)$, Eq. (12) is equivalent to the homogeneous $(\varepsilon=0)$ parabolic reaction-diffusion equation translated to the front reference frame $\left(z=x-\dot{S}_{0} t\right)$ which travels at constant speed $\dot{S}_{0}$. We call $\dot{S}_{0} \equiv c$ and therefore $S_{0}=c t$ where we assume $S(0)=0$. From the solvability condition for the equation at each order of the expansion we will obtain the corresponding corrections to the speed of the front. The solvability integral condition of Eq. (13) is $\int_{-\infty}^{\infty} \psi \mathcal{L}_{1}\left(\phi_{1}\right) d z=0$ [24], where $\psi$ is such that $\mathcal{L}_{1}^{\dagger}(\psi)=0$, $\mathcal{L}_{1}^{\dagger}=\partial_{z z}-\dot{S}_{0} \partial_{z}+f^{\prime}\left(\phi_{0}\right)$ being the self-adjoint operator of $\mathcal{L}_{1}$ [24]. It is easy to show that $\psi=e^{c z} d \phi_{0} / d z$ is an eigenfunction of $\mathcal{L}_{1}^{\dagger}$ with null eigenvalue. The solvability integral condition for Eq. (13) may be written as

$$
\int_{-\infty}^{\infty} e^{c z} \frac{d \phi_{0}}{d z}\left[-\sigma f\left(\phi_{0}\right) \eta_{u}\left(S_{0}\right)-\dot{S}_{1} \frac{d \phi_{0}}{d z}\right] d z=0
$$

so that

$$
\dot{S}_{1}=-\frac{\sigma \eta_{u}(c t) \int_{-\infty}^{\infty} e^{c z} \frac{d \phi_{0}}{d z} f\left(\phi_{0}\right) d z}{\int_{-\infty}^{\infty} e^{c z}\left(\frac{d \phi_{0}}{d z}\right)^{2} d z} .
$$

The integral in the numerator of Eq. (16) may be simplified by using Eq. (12) and integrating by parts

$$
\begin{aligned}
\int_{-\infty}^{\infty} e^{c z} \frac{d \phi_{0}}{d z} f\left(\phi_{0}\right) d z= & -\int_{-\infty}^{\infty} e^{c z} \frac{d \phi_{0}}{d z} \frac{d^{2} \phi_{0}}{d z^{2}} d z \\
& -c \int_{-\infty}^{\infty} e^{c z}\left(\frac{d \phi_{0}}{d z}\right)^{2} d z \\
= & -\frac{1}{2} c \int_{-\infty}^{\infty} e^{c z}\left(\frac{d \phi_{0}}{d z}\right)^{2} d z
\end{aligned}
$$

Finally one can obtain the first correction to the speed

$$
\dot{S}_{1}=\frac{1}{2} \sigma c \eta_{u}(c t)
$$

Note that in Eq. (17) there is no dependence on the solution of $\phi_{0}$ but only on the function $\eta_{u}$.

The speed of the front reads, after inverting the hyperbolic scaling,

$$
v(t)=c+\frac{1}{2} c \eta_{u}(c t \varepsilon) \delta+O\left(\delta^{2}\right)
$$

Before proceeding with the following order in the expansion it is necessary to solve Eq. (13). As $\mathcal{L}_{1}\left(d \phi_{0} / d z\right)=0$ we look for a solution of the form $\phi_{1}(z, t)=\left(d \phi_{0} / d z\right)$ $+\left(d \phi_{0} / d z\right) z F(t)$ in Eq. (13), finding that $F(t)$ $=\frac{1}{2} \sigma \eta_{u}(c t)$. Thus

$$
\phi_{1}(z, t)=\frac{d \phi_{0}}{d z}\left[1+\frac{1}{2} \sigma \eta_{u}(c t) z\right] .
$$

After substituting $S_{0}=c t$, Eqs. (17) and (19) into Eq. (14), and applying the solvability condition [24] $\int_{-\infty}^{\infty} e^{c z}\left(d \phi_{0} / d z\right) \mathcal{L}_{1}\left(\phi_{2}\right) d z=0$ for Eq. (14) we get

$$
\dot{S}_{2}=-\frac{c \sigma^{2}}{8} \eta_{u}(c t)^{2}+\alpha \sigma \eta_{u}^{\prime}(c t)+\frac{c \sigma}{2} \eta_{u}^{\prime}(c t) S_{1}(t)
$$

where $\eta_{u}^{\prime}(c t) \equiv d \eta_{u}(x) /\left.d x\right|_{x=c t}, S_{1}(t)=\frac{1}{2} \sigma \int^{c t} \eta_{u}(x) d x$ [assuming $S(0)=0]$, and

$$
\alpha=-\frac{1}{2}+c \frac{\int_{-\infty}^{\infty} z e^{c z}\left(\frac{d \phi_{0}}{d z}\right)^{2} d z}{\int_{-\infty}^{\infty} e^{c z}\left(\frac{d \phi_{0}}{d z}\right)^{2} d z} .
$$

Note that in this case $\dot{S}_{2}$ depends explicitly on the solution of $\phi_{0}$. In order to compute analytically the second-order correction of the speed it is necessary to have an analytical expression for the zeroth order solution $\phi_{0}(z)$. Some exact solutions are known in the literature [1] for reaction terms of the form $f(\phi)=\phi^{q+1}\left(1-\phi^{q}\right)$ for $q \geqslant 1$. This source term has been applied to forest fires $[25,26]$ and the spread of microorganisms [27]. In this case, the solution for the homogeneous case takes the form

$$
\phi_{0}(z)=\frac{1}{\left(1+e^{b z}\right)^{a}}, \quad c=\frac{1}{\sqrt{1+q}}, \quad b=q c, \quad a=\frac{1}{q} \text {. }
$$


It is easy to check that the integrals involved in Eqs. (16) and (21) are convergent for any $q$. For example, for $q=1$ we have $f(\phi)=\phi^{2}(1-\phi), \alpha=1$, and the speed of the front is given by

$$
\begin{aligned}
v_{S P}(t)= & \dot{S}(t)=\frac{1}{\sqrt{2}}+\frac{\sigma}{2 \sqrt{2}} \eta_{u}\left(\frac{t}{\sqrt{2}}\right) \varepsilon+\left\{-\frac{\sigma^{2}}{8 \sqrt{2}} \eta_{u}\left(\frac{t}{\sqrt{2}}\right)^{2}\right. \\
& \left.+\sigma \eta_{u}^{\prime}\left(\frac{t}{\sqrt{2}}\right)+\frac{\sigma^{2}}{4 \sqrt{2}} \eta_{u}^{\prime}\left(\frac{t}{\sqrt{2}}\right) \int^{t / \sqrt{2}} \eta_{u}(x) d x\right\} \varepsilon^{2} \\
& +O\left(\varepsilon^{3}\right),
\end{aligned}
$$

where we have made use of Eqs. (17) and (20).

For mathematical and numerical simplicity let us illustrate the above results for the case where $\eta_{u}(x)=x$ is linear. Taking $\sigma=1$ we have from Eq. (22) $v(t)=1 / \sqrt{2}+t \varepsilon / 4+\varepsilon^{2}$ and inverting the hyperbolic scaling we obtain, for $t \ll O\left(\varepsilon^{-2}\right)$,

$$
v_{S P}(t)=\frac{1}{\sqrt{2}}+\left(1+\frac{t}{4}\right) \varepsilon^{2}+O\left(\varepsilon^{3}\right),
$$

where the subscript $S P$ stands for "singular perturbation" result. For any non-KPP $f(\phi)$ one has

$$
v_{S P}(t)=c+\left(\frac{c^{2} t}{2}+\alpha\right) \varepsilon^{2}+O\left(\varepsilon^{3}\right) .
$$

The local speed approach assumes that the front position changes adiabatically in time as the front moves into a region where the characteristic parameters $D$ and $U$ change. For the first of Eqs. (1) and for a source term $f(\phi)=\phi^{2}(1-\phi)$ the speed of the front is locally given by $v=\sqrt{D U\left(\varepsilon x_{f}\right) / 2}$, where $x_{f}$ is the position of the front. To be more specific, let us take also $\eta_{u}(x)=x$ and $\sigma=1$. In consequence, the dependence of speed of the front on the time is obtained by integrating the differential equation

$$
\frac{d x_{f}}{d t}=\sqrt{\frac{1+\varepsilon^{2} x_{f}}{2}}
$$

for the position of the front in dimensionless units. Taking $x_{f}(0)=0$ the local speed approach yields, for this case,

$$
v_{L A}(t)=\frac{1}{\sqrt{2}}+\frac{t \varepsilon^{2}}{4},
$$

where the subscript $L A$ stands for "local approach." In Fig. 1 we compare, for different values of $\varepsilon$, the numerical results for the front speed of the first equation in Eqs. (1) for $U(\varepsilon x)=1+\delta \eta_{u}(\varepsilon x), \eta_{u}(x)=x$, and $\phi(x, 0)$, a Heaviside function, to the analytical solutions (23) and (26). We observe that $v_{S P}$ is in better agreement with numerical solutions than $v_{L A}$, after an initial transient. This transient is due to two factors: it takes a certain interval of time for the front to fully develop and the asymptotic approximation is valid for $\eta_{u}(x)=O(1)$.

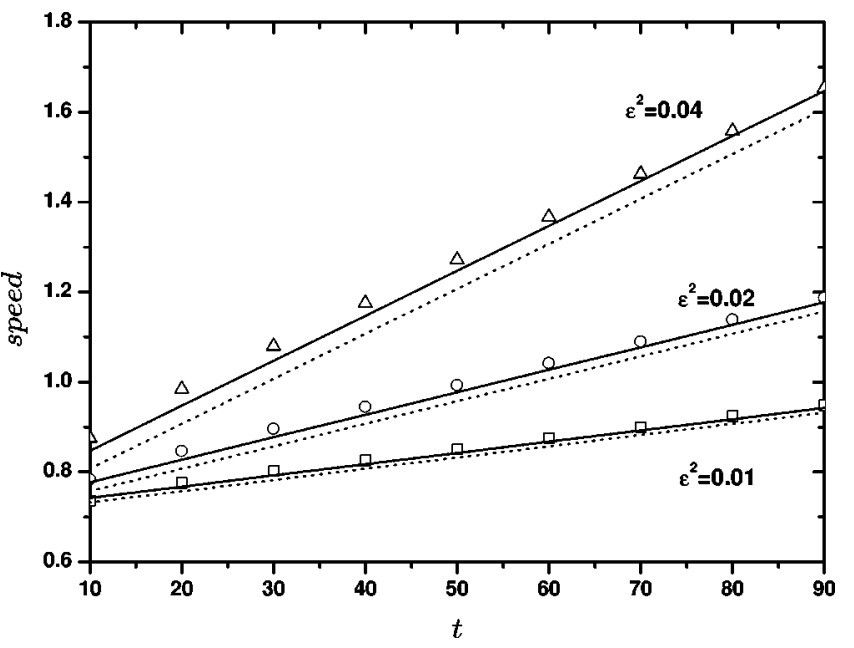

FIG. 1. Comparison of the temporal evolution of the speed of fronts (in dimensionless units) between the singular perturbation analytical result given in Eq. (23) (solid lines), the local speed approach (26) (dashed lines), and the numerical results (symbols) for different values of $\varepsilon$. This is the case $\eta_{u}(x)=x$ and $f=\phi^{2}(1$ $-\phi$ ) for nonuniform reaction rate.

\section{B. Hamilton-Jacobi dynamics}

The use of the Hamilton-Jacobi dynamics to study the front propagation is initially devoted to Freidlin [28] who treated the KPP minimal speed for slowly varying media using probabilistic (large deviation) approach but also rigorous mathematics has done by Gärtner [29] and Evans [30]. However, as we will show in the last section, it is not necessary to assume either smooth or weak heterogeneities. We stress that singular perturbation analysis (preceding section) does not yield a fully analytical result for the very important KPP kinetic $f(\phi)=\phi(1-\phi)[1,31]$ if one needs to go beyond first order in $\delta$, because the exact unperturbed solution is unknown and the solvability integrals diverge. In this section we determine the temporal evolution of the position of the reaction front for the logistic case. We replace $\phi(x / \varepsilon, t / \varepsilon)$ in Eq. (3) by an auxiliary field $G(x, t) \geqslant 0$ through the exponential transformation

$$
\phi(x, t)=e^{-G(x, t) / \varepsilon} .
$$

We expect that $\phi(x, t)$ tends to a unit step function as $\varepsilon$ $\rightarrow 0$. The equality $G(x, t)=0$ determines the position of the front. Substituting Eq. (27) into Eq. (2), for the KPP kinetics $f(\phi)=\phi(1-\phi)$, we get, to leading order, the equation $(\varepsilon$ $=0$ )

$$
\partial_{t} G+\left(\partial_{x} G\right)^{2}+U(\delta, x)=0
$$

for the action functional where $U(\delta, x)=1+\delta \eta_{u}(x)$. From the analogy with the Hamilton-Jacobi equation $\partial_{t} G$ $+H\left(\partial_{x} G, x\right)=0$, we define the Hamiltonian

$$
H=p^{2}+1+\delta \eta_{u}(x)
$$


where $p \equiv \partial_{x} G$ plays the role of conjugated momenta of $x$. Equation (28) may be solved by using the Hamilton equations

$$
\begin{gathered}
\frac{d x}{d s}=\partial_{p} H=2 p, \\
\frac{d p}{d s}=-\partial_{x} H=-\delta \frac{d \eta_{u}}{d x},
\end{gathered}
$$

from which we get the differential equation for $x(s)$

$$
\ddot{x}(s)+2 \delta \frac{d \eta_{u}[x(s)]}{d x}=0
$$

under the boundary conditions

$$
\begin{aligned}
& x(s=0)=x, \\
& x(s=t)=0 .
\end{aligned}
$$

The solution for the action functional $G(x, t)$ is given by

$$
G(x, t)=\min _{x(s=0)=x, x(s=t)=0} \int_{0}^{t} L[x(s), p(s)] d s,
$$

where $L[x(s), p(s)]$ is the Lagrangian function defined by $L[x(s), p(s)]=p(s) \dot{x}(s)-H$. As the Hamiltonian function does not depend explicitly on the time $s$ there exists the energy integral

$$
\frac{\dot{x}(s)^{2}}{4}+1+\delta \eta[x(s)]=E,
$$

and therefore, from Eq. (32) one has

$$
G(x, t)=-E t+\frac{1}{2} \int_{0}^{t} \dot{x}(s)^{2} d s .
$$

Let us now detail the calculations of two specific and simple choices of $\eta_{u}$ where Eq. (30) has exact solution. The first one is $\eta_{u}(x)=x$ as in the preceding section. In this case Eq. (30) yields, together with conditions (31),

$$
x(s)=x-\delta s^{2}-\frac{s x}{t}+\delta s t, \quad \text { for } 0 \leqslant s \leqslant t
$$

and

$$
E=1+\frac{1}{2} x \delta+\frac{1}{4} \delta^{2} t^{2}+\frac{x^{2}}{4 t^{2}}
$$

Finally, from Eq. (34)

$$
G(x, t)=-t-\frac{1}{12} \delta^{2} t^{3}-\frac{1}{2} x t \delta+\frac{x^{2}}{4 t} .
$$

The position of the front given by $G(x, t)=0$ is

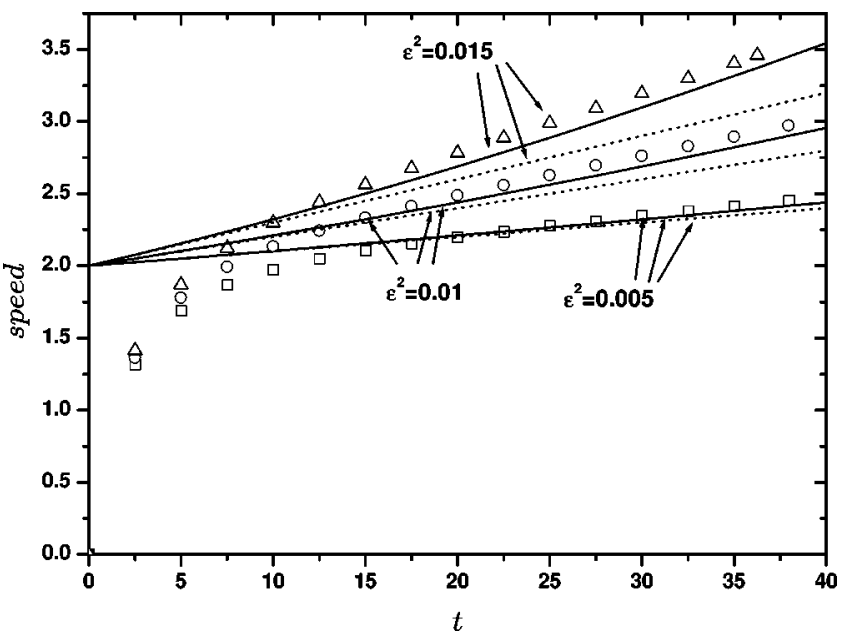

FIG. 2. Comparison of the temporal evolution of the speed of fronts (in dimensionless units) between the Hamilton-Jacobi analytical result given in Eq. (36) (solid lines), the local speed approach (38) (dashed lines), and the numerical results (symbols) for different values of $\varepsilon$. This is the case $\eta_{u}(x)=x$ and $f=\phi(1-\phi)$ for nonuniform reaction rate.

$$
x(t)=\delta t^{2}+2 t \sqrt{1+\frac{1}{3} \delta^{2} t^{2}}
$$

and the exact relationship for the speed after inverting the hyperbolic scaling is

$$
v_{H J}(t)=\frac{d x}{d t}=2 \delta \varepsilon t+\frac{4 \varepsilon^{2} \delta^{2} t^{2}+6}{\sqrt{3 \varepsilon^{2} \delta^{2} t^{2}+9}}
$$

for any $\delta$. For weak inhomogeneities $(\delta \ll 1)$ one has for $\sigma$ $=1$, in Eq. (36),

$$
v_{H J}(t) \simeq 2+2 t \varepsilon^{2}+t^{2} \varepsilon^{4}+O\left(\varepsilon^{8}\right),
$$

which holds only for $t \ll O\left(\varepsilon^{-2}\right)$. The subscript $H J$ stands for "Hamilton-Jacobi" result.

The local speed approach for the KPP kinetics yields $v$ $=2 \sqrt{D U\left(\varepsilon x_{f}\right)}$ and the differential equation for the position of the front is

$$
\frac{d x_{f}}{d t}=2 \sqrt{1+\varepsilon^{2} x_{f}},
$$

which after integrating under the initial condition $x_{f}(0)=0$ may be written as

$$
v_{L A}(t)=2+2 t \varepsilon^{2} .
$$

In Fig. 2 we compare Eqs. (36) and (38) with the numerical solution for the first equation in Eq. (1) for different values of $\varepsilon$. After the initial transient, we observe, in general, good agreement. However, $v_{H J}$ is in better agreement with numerical solutions than $v_{L A}$, after the initial transient.

Another case with exact solution is $\eta_{u}(x)=x^{2}$. In this case the Hamiltonian is the same as for the simple harmonic oscillator. Equation (30) with Eq. (31) yields 
$x(s)=x\left[\cos (2 \sqrt{\delta} s)-\frac{\cos (2 \sqrt{\delta} t)}{\sin (2 \sqrt{\delta} t)} \sin (2 \sqrt{\delta} s)\right]$ for $0 \leqslant s \leqslant t$.

The energy integral and action functional are

$$
\begin{gathered}
E=1+\frac{\delta x^{2}}{\sin ^{2}(2 \sqrt{\delta} t)}, \\
G(x, t)=-t+\frac{\sqrt{\delta} x^{2} \cos (2 \sqrt{\delta} t)}{2 \sin (2 \sqrt{\delta} t)}
\end{gathered}
$$

and the position of the front is given by

$$
x(t)=\sqrt{\frac{2 t \sin (2 \sqrt{\delta} t)}{\sqrt{\delta} \cos (2 \sqrt{\delta} t)}} .
$$

From Eq. (39) one can see that the position as well as the speed of the front takes the infinite value just when $t$ $=\pi / 4 \sqrt{\delta}$. However, for weak heterogeneities $(\delta \ll 1)$ one has

$$
v_{H J}(t)=2+4 t^{2} \delta \varepsilon^{2}+76 / 9 t^{4} \varepsilon^{4} \delta^{2}+O\left(\delta^{3}\right),
$$

which does not have singularities but is valid only for $t$ $\ll O\left(\varepsilon^{-1}\right)$.

If we assume weak heterogeneities $(\delta \ll 1)$ we can approximate the speed of the front for any general $\eta(x)$. Details of the calculations are given in the appendix. It is important to note that Eq. (A7) is, up to $\delta$ order, equal to Eq. (18) for the KPP kinetic term where $c=2$ but differs for higher orders. For $\eta_{u}(x)=x$ and $x^{2}$, from Eq. (A7) we recover Eqs. (37) and (40), respectively.

\section{NONUNIFORM DIFFUSION}

We consider now the problem

$$
\begin{gathered}
\varepsilon \partial_{t} \phi=\varepsilon^{2} \partial_{x}\left[D(x) \partial_{x} \phi\right]+f(\phi), \\
D(x) \equiv 1+\delta \eta_{D}(x),
\end{gathered}
$$

where $\delta$ is the amplitude of the heterogeneities and $\eta_{D}(x)$ is the heterogeneity function in diffusion. In this case, the local speed approach yields the same speed as for nonuniform reaction rate if $\eta_{D}(x)=\eta_{u}(x)$. As we will see below, this result is in disagreement with the singular perturbative and the Hamilton-Jacobi results.

\section{A. Singular perturbation analysis}

As in the preceding section, we assume $\delta \equiv \sigma \varepsilon$ in Eq. (41) where $\sigma=O(1)$ and we assume the existence of an outer and a boundary layer region. Equation (41), together with the corresponding boundary conditions, becomes

$$
\begin{gathered}
\varepsilon \partial_{t} \phi=\varepsilon^{2} \partial_{x x} \phi+\varepsilon^{3}\left[\frac{d \eta_{D}}{d x} \partial_{x} \phi+\eta_{D}(x) \partial_{x x} \phi\right]+f(\phi), \\
\lim _{x \rightarrow-\infty} \phi=1, \quad \lim _{x \rightarrow \infty} \phi=0 .
\end{gathered}
$$

In order to solve Eq. (42) in the outer region we use expansion (5). We can easily see that the effect of the het- erogeneity in the coefficient of diffusion does not affect the solution at least until $O\left(\varepsilon^{3}\right)$. Thus $\phi(x, t ; \varepsilon)=O\left(\varepsilon^{3}\right)$ to the left of the boundary layer and $\phi(x, t ; \varepsilon)=1+O\left(\varepsilon^{3}\right)$ to the right of the boundary layer.

In order to study the dynamics of Eq. (42) inside the boundary layer we substitute Eqs. (9)-(11) into Eq. (42) and collect terms with equal powers of $\varepsilon$. We obtain Eq. (12) for the lowest order, and

$$
\begin{gathered}
\mathcal{L}_{1}\left(\phi_{1}\right)=-\partial_{z z} \phi_{0} \sigma \eta_{D}\left(S_{0}\right)-\dot{S}_{1} \partial_{z} \phi_{0}, \\
\mathcal{L}_{1}\left(\phi_{2}\right)=-\dot{S}_{2} \partial_{z} \phi_{0}-\dot{S}_{1} \partial_{z} \phi_{1}-\frac{1}{2} f^{\prime \prime}\left(\phi_{0}\right) \phi_{1}^{2} \\
-\sigma \eta_{D}\left(S_{0}\right) \partial_{z z} \phi_{1}-\sigma \eta_{D}^{\prime}\left(S_{0}\right)\left(z+S_{1}\right) \partial_{z z} \phi_{0} \\
-\sigma \eta_{D}^{\prime}\left(S_{0}\right) \partial_{z} \phi_{0}+\partial_{t} \phi_{1}
\end{gathered}
$$

for first and second orders, respectively. From the solvability conditions and assuming $\phi=\phi_{0}(z)$ one has $\dot{S}_{0}=c$ constant and

$$
\begin{gathered}
\dot{S}_{1}=\frac{1}{2} c \sigma \eta_{D}(c t), \\
\dot{S}_{2}=-\frac{c}{8} \sigma^{2} \eta_{D}(c t)^{2}-\frac{\sigma}{2} \eta_{D}^{\prime}(c t)+\frac{c^{2} \sigma^{2}}{4} \eta_{D}(c t) \eta_{D}^{\prime}(c t), \\
\phi_{1}(z, t)=\frac{d \phi_{0}}{d z}\left[1-\frac{1}{2} \eta_{D}(c t) \sigma z\right] .
\end{gathered}
$$

The speed of the front is finally given by

$$
\begin{aligned}
v_{S P}(t)= & c+\frac{1}{2} c \sigma \eta_{D}(c t) \varepsilon+\left[-\frac{c}{8} \sigma^{2} \eta_{D}(c t)^{2}-\frac{\sigma}{2} \eta_{D}^{\prime}(c t)\right. \\
& \left.+\frac{c^{2} \sigma^{2}}{4} \eta_{D}(c t) \eta_{D}^{\prime}(c t)\right] \varepsilon^{2}+O\left(\varepsilon^{3}\right)
\end{aligned}
$$

It is very interesting to note that, in this case, up to second order in $\varepsilon$ the speed correction does not depend on the solution of $\phi_{0}$. For $\eta_{D}(x)=x, \sigma=1$, and general non-KPP kinetic term one has, for $t \ll O\left(\varepsilon^{-2}\right)$,

$$
v_{S P}(t)=c+\left(\frac{c^{2} t}{2}-\frac{1}{2}\right) \varepsilon^{2}+O\left(\varepsilon^{3}\right)
$$

after inverting the hyperbolic scaling. In Fig. 3 we compare Eq. (44) for $f=\phi^{2}(1-\phi)$ and Eq. (26) with the numerical solution of the second equation in Eqs. (1) with $D(\varepsilon x)=1$ $+\delta \eta_{D}(\varepsilon x)$ and $\eta_{D}(x)=x$ for different values of $\varepsilon$. In this case $v_{L A}$ is in slightly better agreement with numerical solutions than $v_{S P}$, contrary to the previous case.

\section{B. Hamilton-Jacobi dynamics}

The Hamilton-Jacobi equation for problem (41), to leading order $(\varepsilon=0)$, with a KPP kinetic term is 


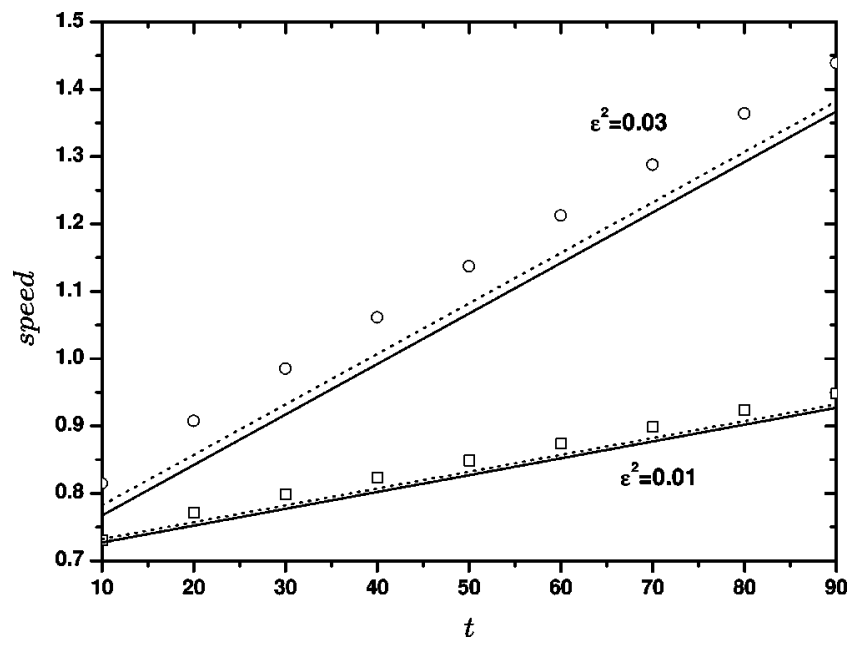

FIG. 3. Comparison of the temporal evolution of the speed of fronts (in dimensionless units) between the singular perturbed analytical result given in Eq. (44) for $c=1 / \sqrt{2}$ (solid lines), the local speed approach (26) (dashed lines), and the numerical results (symbols) for different values of $\varepsilon$. This is the case $\eta_{D}(x)=x$ and $f$ $=\phi^{2}(1-\phi)$ for nonuniform diffusion coefficient.

$$
\partial_{t} G+\left[1+\delta \eta_{D}(x)\right]\left(\partial_{x} G\right)^{2}+1=0 .
$$

The Hamilton equations are

$$
\begin{gathered}
\frac{d x}{d s}=\partial_{p} H=2\left[1+\delta \eta_{D}(x)\right] p, \\
\frac{d p}{d s}=-\partial_{x} H=-\delta p^{2} \frac{d \eta_{D}}{d x} .
\end{gathered}
$$

The equation for $x(s)$ is

$$
\ddot{x}(s)-\frac{\delta \dot{x}(s)^{2}}{2\left(1+\delta \eta_{D}[x(s)]\right)} \frac{d \eta_{D}}{d x}[x(s)]=0,
$$

the corresponding integral energy is

$$
\frac{\dot{x}(s)^{2}}{4\left(1+\delta \eta_{D}[x(s)]\right)}+1=E
$$

and the action functional is

$$
G(x, t)=-E t+\frac{1}{2} \int_{0}^{t} \frac{\dot{x}(s)^{2}}{1+\delta \eta_{D}[x(s)]} d s .
$$

Let us now be more specific for the two cases where Eq. (46) has exact solution. First we take $\eta_{D}(x)=x$. From Eqs. (31) and (46) one has

$$
\begin{aligned}
& x(s)=x+\frac{s^{2}}{\delta t^{2}}(\sqrt{1+\delta x}-1)^{2}-\frac{2 s}{\delta t}(1+\delta x-\sqrt{1+\delta x}) \\
& \quad \text { for } 0 \leqslant s \leqslant t
\end{aligned}
$$

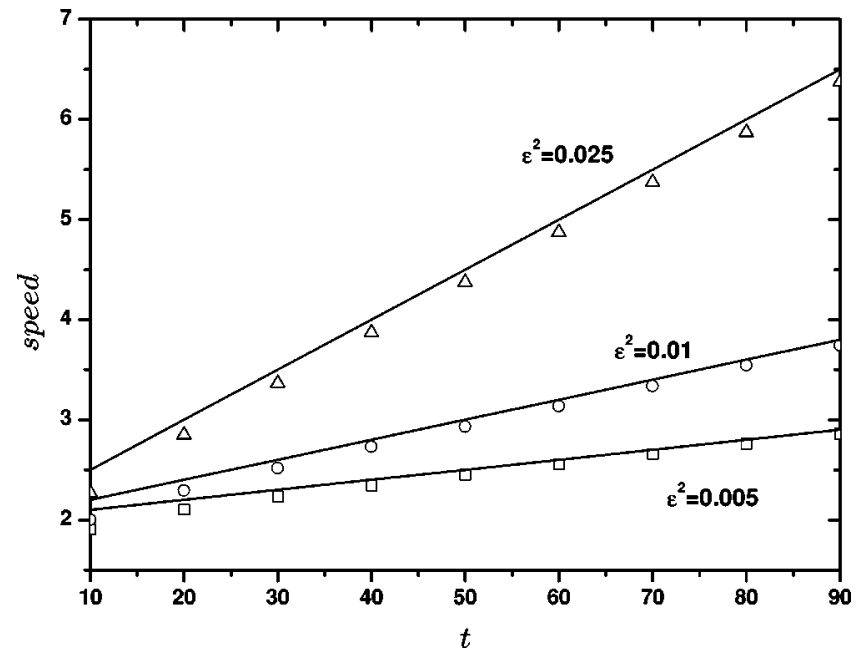

FIG. 4. Comparison of the temporal evolution of the speed of fronts (in dimensionless units) between the Hamilton-Jacobi analytical result given in Eq. (49) (solid lines), the local speed approach (38) (dashed lines), and the numerical results (symbols) for different values of $\varepsilon$. This is the case $\eta_{D}(x)=x$ and $f=\phi(1-\phi)$ for nonuniform diffusion coefficient.

$$
E=1+\frac{1}{\delta^{2} t^{2}}(\sqrt{1+\delta x}-1)^{2}
$$

and from Eq. (48) one gets

$$
G(x, t)=\frac{1}{\delta^{2} t}(\sqrt{1+\delta x}-1)^{2}-t .
$$

Therefore the speed is given, in an exact form, by

$$
v_{H J}(t)=2+2 t \delta \varepsilon
$$

after inverting the hyperbolic scaling. Note first of all that this result is equal to that obtained from the local speed approach. As in Sec. II, note that the result obtained in this section is essentially a leading order approximation while the result obtained in Sec. III A is an $O(\varepsilon)$ approximation.

In Fig. 4 we compare Eqs. (49) (taking $\delta=\varepsilon$ ) and (38) with the numerical result of the second equation in Eqs. (1). In this case the agreement between both analytical methods and the numerical results is not so good as for nonuniform reaction rate.

From the local speed approach the speed for a given dependence of the reaction rate on the spatial coordinate and constant diffusion coefficient is equal to the speed when the reaction rate is constant and the diffusion coefficient depends on the spatial coordinate with the same functional dependence as the above reaction rate. We have checked numerically for some values of $\varepsilon$ that the speed for the linear dependence of the reaction rate is not equal, although very similar, to the speed for the linear dependence of the diffusion coefficient.

From the singular perturbative analysis and the HamiltonJacobi methods we can conclude that for the problems $\partial_{t} \phi=\partial_{x x} \phi+\left[1+\delta \eta_{u}(\varepsilon x)\right] f(\phi) \quad$ and $\quad \partial_{t} \phi=\partial_{x}\{[1$ 
$\left.\left.+\delta \eta_{D}(\varepsilon x)\right] \partial_{x} \phi\right\}+f(\phi)$ with non-KPP $f(\phi)$, such that $f(0)$ $=f(1)=0$, where $\delta, \varepsilon \ll 1$ (weak and smooth heterogeneities) and $\eta_{u, D}(x)$ is a continuous and derivable function, the speed of the front has the formal solution

$$
v(t)=c+\frac{1}{2} c \eta_{u, D}(c t \varepsilon) \delta+O\left(\delta^{2}\right),
$$

for $t \ll O\left(\varepsilon^{-1}\right)$ where $c$ is the asymptotic (constant) speed for the homogeneous problem $\partial_{t} \phi=\partial_{x x} \phi+f(\phi)$. If $\eta$ is an increasing/decreasing function of space, the front is accelerated/decelerated.

\section{FRACTAL MEDIA}

In this section we illustrate the advantages of using the Hamilton-Jacobi method for dealing with heterogeneous media. In particular, we get an exact expression for the front speed propagation in fractal media. The reaction-diffusion process in a fractal may be described by the equation for the probability density of O'Shaughnessy and Procaccia [32] coupled to a KPP kinetic term

$$
\partial_{t} \phi=\frac{1}{r^{d-1}} \partial_{r}\left(D r^{d-1-\theta} \partial_{r} \phi\right)+U \phi(1-\phi),
$$

where $d$ is the dimension of the fractal, $\theta$ is an index which is 0 for the classical normal situation (Euclidean media), and $D$ is a kind of diffusion coefficient. After taking into account the hyperbolic scaling $r \rightarrow r / \varepsilon$ and $t \rightarrow t / \varepsilon$ and the field $G^{\varepsilon}(r, t)=-\varepsilon \ln \phi(r, t)$, one has from Eq. (50),

$$
\begin{aligned}
-\partial_{t} G^{\varepsilon}= & -\varepsilon^{1+\theta} D(d-1-\theta) r^{-1-\theta} \partial_{r} G^{\varepsilon}+\varepsilon^{\theta} D r^{-\theta}\left(\partial_{r} G^{\varepsilon}\right)^{2} \\
& -\varepsilon^{1+\theta} D r^{-\theta} \partial_{r r} G^{\varepsilon}+U .
\end{aligned}
$$

The first and third terms in the right-hand side of Eq. (51) have the same order of magnitude and in the asymptotic limit $(\varepsilon \rightarrow 0)$ both terms may be neglected in front of the second term and in consequence, the Hamilton-Jacobi for the front propagation in a fractal media is

$$
\partial_{t} G+(\varepsilon / r)^{\theta} D\left(\partial_{r} G^{\varepsilon}\right)^{2}+U=0,
$$

where $G(r, t)=\lim _{\varepsilon \rightarrow 0} G^{\varepsilon}(r, t)$. Proceeding as in the above sections one has

$$
G(r, t)=\frac{r^{2+\theta}}{t D \varepsilon^{\theta}(2+\theta)^{2}}-U t
$$

and the exact expression for the speed of the front, once the hyperbolic scaling is inverted, is

$$
v(t)=2\left[\frac{D U}{(2+\theta)^{\theta} t^{\theta}}\right]^{1 /(2+\theta)},
$$

which describes a decelerated front. In Fig. 5 we compare the exact result (52) with the results of the numerical solutions of Eq. (50) for different values of $\theta$. We stress that in this case of physical interest, in contrast to what would

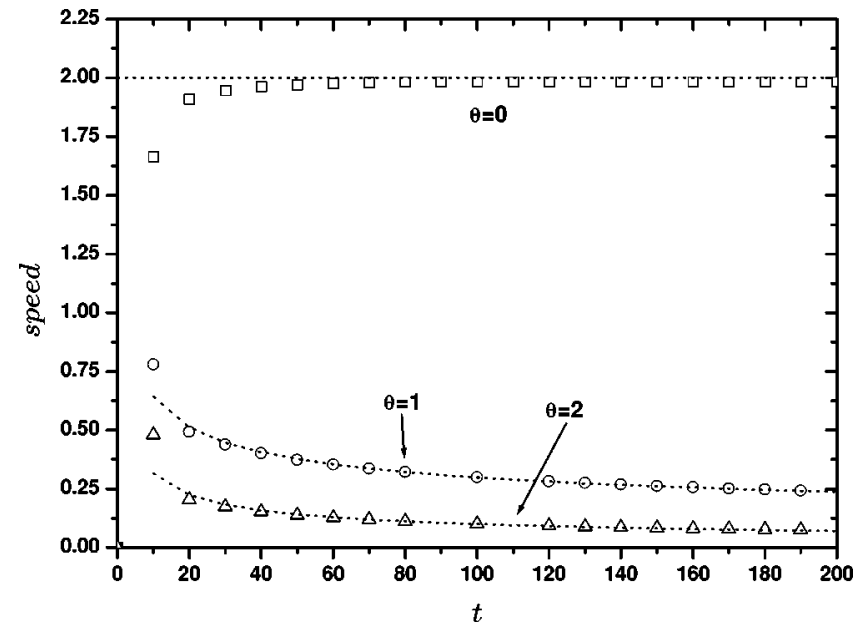

FIG. 5. Comparison of the temporal evolution of the speed of fronts (in dimensionless units) derived from the Hamilton-Jacobi method (52) (dashed lines) with numerical solutions of Eq. (50) (symbols) for fractal media. For $\theta=0$ one recovers the Fisher result. We have taken $D=U=d=1$.

happen if using the local approach, we have not assumed weak or smooth heterogeneities because we have applied the Hamilton-Jacobi method.

\section{CONCLUSIONS}

We have studied how deterministic heterogeneities influence the front speed of parabolic reaction-diffusion equations where the reaction term and/or the diffusion coefficient depend on the spatial coordinates. We have derived analytic expressions for the speed of fronts that are valid for initially fully developed fronts or for more general initial conditions in the asymptotic limit. The singular perturbative analysis and the geometrical method of Hamilton-Jacobi have been employed to find the speed of the fronts propagating in deterministic heterogeneous media. The singular perturbative analysis has been used when spatial heterogeneities are weak $(\delta \ll 1)$ and smooth $(\varepsilon \ll 1)$ and may be successfully applied only for fronts with non-KPP kinetics (pushed fronts). The expressions obtained for the speed are power series of $\varepsilon$, where secular terms appear and in consequence are not uniformly valid in time. However, for the simplest case of linear heterogeneities these expressions have been compared to numerical solutions exhibiting a good agreement.

The Hamilton-Jacobi method we used here only holds for fronts with KPP kinetics (pulled fronts). However, this method allows us to work without assuming either smooth or weak heterogeneities. We have compared the results for the simplest case of linear heterogeneities with numerical solutions and a good agreement is found again. Exact solution for the speed of fronts traveling in fractal media is obtained and compared to numerical solutions. We have found an excellent agreement and it has been shown both analytically and numerically that the front is decelerated.

Finally, the local speed approach has been compared with the above analytical methods and numerical solutions. For nonuniform diffusion coefficient this approach slightly im- 
proves the singular perturbative method and yields the same result found with the Hamilton-Jacobi method. However, for nonuniform reaction rate both singular perturbative and Hamilton-Jacobi are in better agreement with numerical results than the local speed approach.

\section{ACKNOWLEDGMENTS}

This work was partially funded by the Generalitat de Catalunya under Grant No. SGR-2001-00186 (V.M. and J.F.), by the MICYT under Grant Nos. REN-2000-1621 CLI (J.F.) and BFM-2000-0351 (V.M. and J.F.), and by the Burroughs Wellcome Fund (HR).

\section{APPENDIX: WEAK HETEROGENEITIES IN HAMILTON-JACOBI DYNAMICS}

In this appendix we develop the calculations to obtain the speed of fronts for weak heterogeneities $(\delta \ll 1)$ for both nonuniform reaction and nonuniform diffusion.

\section{Nonuniform reaction rate}

The first step is to solve Eq. (30) under Eq. (31) by using regular perturbation analysis. By substituting the expansion $x(s)=x_{0}(s)+\delta x_{1}(s)+\delta^{2} x_{2}(s)+O\left(\delta^{3}\right)$ into Eq. (30) one has $\ddot{x}_{0}(s)=0$ for the lowest order,

$$
\ddot{x}_{1}(s)=-2 \frac{d \eta_{u}\left[x_{0}(s)\right]}{d x}
$$

for the first order, and

$$
\ddot{x}_{2}(s)=-2 x_{1}(s) \frac{d^{2} \eta_{u}\left[x_{0}(s)\right]}{d x^{2}}
$$

for the second order. From Eqs. (31) $x_{0}(0)=x, x_{0}(t)=0$ and $x_{i}(0)=x_{i}(t)=0$ for $i \geqslant 1$ one obtains for $0 \leqslant s \leqslant t$,

$$
\begin{gathered}
x_{0}(s)=x-x s / t, \\
x_{1}(s)=\frac{2 t}{x} \int \eta_{u}(x-x s / t) d s-\frac{2 t s}{x^{2}} \int_{0}^{x} \eta_{u}\left(x^{\prime}\right) d x^{\prime}+\frac{2 t^{2}}{x^{2}} \int^{x} \eta_{u}\left(x^{\prime}\right) d x^{\prime}, \\
x_{2}(s)=-\frac{4 t^{4}}{x^{4}} \eta_{u}(x-x s / t) \int^{x} \eta_{u}\left(x^{\prime}\right) d x^{\prime}-\frac{8 t^{3}}{x^{4}} \int^{x} \eta_{u}\left(x^{\prime}\right) d x^{\prime} \int \eta_{u}(x-x s / t) d s+\frac{4 t^{3}}{x^{4}} s \eta_{u}(x-x s / t) \\
-\frac{4 t^{3}}{x^{3}} \eta_{u}(x-x s / t) \int \eta_{u}(x-x s / t) d s+\frac{6 t^{3}}{x^{3}} \int \eta_{u}^{2}(x-x s / t) d s+\frac{8 t^{3} s}{x^{5}}\left[\int_{0}^{x} \eta_{u}\left(x^{\prime}\right) d x^{\prime}\right]^{2} \\
-\frac{6 t^{3} s}{x^{4}} \int_{0}^{x} \eta_{u}^{2}(x-x s / t) d s-\frac{8 t^{4}}{x^{5}} \int^{x} \eta_{u}\left(x^{\prime}\right) d x^{\prime}+\frac{6 t^{4}}{x^{4}} \int \eta_{u}^{2}\left(x^{\prime}\right) d x^{\prime} .
\end{gathered}
$$

The energy integral (33) reads

$$
E=1+\frac{x^{2}}{4 t^{2}}+\frac{\delta}{x} \int_{0}^{x} \eta_{u}\left(x^{\prime}\right) d x^{\prime}+O\left(\delta^{2}\right)
$$

and from Eq. (34),

$$
G(x, t)=\frac{x^{2}}{4 t}-t-\frac{\delta t}{x} \int_{0}^{x} \eta_{u}\left(x^{\prime}\right) d x^{\prime}+\delta^{2} \frac{t^{3}}{x^{4}}\left\{\left[\int_{0}^{x} \eta_{u}\left(x^{\prime}\right) d x^{\prime}\right]^{2}-x \int_{0}^{x} \eta_{u}^{2}\left(x^{\prime}\right) d x^{\prime}\right\}+O\left(\delta^{3}\right) .
$$

The position $x=x(t)$ of the front comes from $G(x, t)=0$ which has to be solved, by using the expansion $x(t)=x_{0}(t)$ $+\delta x_{1}(t)+\delta^{2} x_{2}(t)+O\left(\delta^{3}\right)$, to obtain

$$
x(t)=2 t+\frac{\delta}{2} \int_{0}^{2 t} \eta_{u}\left(x^{\prime}\right) d x^{\prime}+\frac{\delta^{2}}{4}\left\{\eta_{u}(2 t) \int_{0}^{2 t} \eta_{u}\left(x^{\prime}\right) d x^{\prime}+\frac{1}{2} \int_{0}^{2 t} \eta_{u}^{2}\left(x^{\prime}\right) d x^{\prime}-\frac{1}{t}\left[\int_{0}^{2 t} \eta_{u}\left(x^{\prime}\right) d x^{\prime}\right]^{2}\right\}+O\left(\delta^{3}\right)
$$

and the speed is 


$$
\begin{aligned}
v(t)= & +\delta \eta_{u}(2 t \varepsilon)+\delta^{2}\left\{\frac{1}{4 \varepsilon^{2} t^{2}}\left[\int_{0}^{2 t \varepsilon} \eta_{u}\left(x^{\prime}\right) d x^{\prime}\right]^{2}-\frac{\eta_{u}(2 t \varepsilon)}{t \varepsilon} \int_{0}^{2 t \varepsilon} \eta_{u}\left(x^{\prime}\right) d x^{\prime}+\frac{3}{4} \eta_{u}^{2}(2 t \varepsilon)+\left.\frac{1}{2} \frac{d \eta_{u}}{d x}\right|_{x=2 t \varepsilon} \int_{0}^{2 t \varepsilon} \eta_{u}\left(x^{\prime}\right) d x^{\prime}\right\} \\
& +O\left(\delta^{3}\right)
\end{aligned}
$$

once the hyperbolic scaling is inverted.

\section{Nonuniform diffusion coefficient}

Proceeding analogously as in the preceding section the perturbed solution to Eq. (46) is given by

$$
\begin{gathered}
x_{0}(s)=x-x s / t \\
x_{1}(s)=-\frac{x}{2 t} \int \eta_{D}(x-x s / t) d s+\frac{s}{2 t} \int_{0}^{x} \eta_{D}\left(x^{\prime}\right) d x^{\prime}-\frac{1}{2} \int^{x} \eta_{D}\left(x^{\prime}\right) d x, \\
x_{2}(s)=\frac{s}{4 t} \eta_{D}(x-x s / t) \int_{0}^{x} \eta_{D}\left(x^{\prime}\right) d x^{\prime}-\frac{1}{4} \eta_{D}(x-x s / t) \int^{x} \eta_{D}\left(x^{\prime}\right) d x-\frac{x}{4 t} \eta_{D}(x-x s / t) \int \eta_{D}(x-x s / t) d s \\
+\frac{3 x}{8 t} \int \eta_{D}^{2}(x-x s / t) d s-\frac{3 s}{8 t} \int_{0}^{x} \eta_{D}^{2}\left(x^{\prime}\right) d x^{\prime}+\frac{3}{8} \int^{x} \eta_{D}^{2}\left(x^{\prime}\right) d x,
\end{gathered}
$$

for $0<s<t$, and the energy integral and the action functional are

$$
\begin{gathered}
E=1+\frac{x^{2}}{4 t^{2}}+\frac{\delta}{x} \int_{0}^{x} \eta_{D}\left(x^{\prime}\right) d x^{\prime}+\delta^{2}\left[\frac{t^{2}}{x^{4}}\left(\int_{0}^{x} \eta_{D}\left(x^{\prime}\right) d x^{\prime}\right)^{2}+\frac{3 x}{16 t^{2}} \int_{0}^{x} \eta_{D}^{2}\left(x^{\prime}\right) d x^{\prime}\right]+O\left(\delta^{3}\right), \\
G(x, t)=-t+\frac{x^{2}}{4 t}+\frac{\delta t}{x} \int_{0}^{x} \eta_{D}\left(x^{\prime}\right) d x^{\prime}+\delta^{2}\left[\frac{t^{3}}{x^{4}}\left(\int_{0}^{x} \eta_{D}\left(x^{\prime}\right) d x^{\prime}\right)^{2}+\frac{3 x}{16 t} \int_{0}^{x} \eta_{D}^{2}\left(x^{\prime}\right) d x^{\prime}\right]+O\left(\delta^{3}\right) .
\end{gathered}
$$

Finally, from the temporal derivative of the position of the front given by $G=0$ one gets

$$
v(t)=2+\eta_{D}(2 t \varepsilon) \delta+\delta^{2}\left[\left.\frac{1}{2} \frac{d \eta_{D}}{d x}\right|_{x=2 t \varepsilon} \int_{0}^{2 t \varepsilon} \eta_{D}\left(x^{\prime}\right) d x^{\prime}-\frac{1}{4} \eta_{D}^{2}(2 t \varepsilon)\right]+O\left(\delta^{3}\right) .
$$

[1] J.D. Murray, Mathematical Biology (Springer-Verlag, Berlin, 1989).

[2] S.K. Scott, Oscillations, Waves and Chaos in Chemical Kinetics (Oxford University Press, Oxford, 1994).

[3] A.N. Kolmogorov, I.G. Petrovskii, and N.S. Piskunov, Mosc. Univ. Bull. Math. Ser. A 1, 1 (1937).

[4] R.A. Fisher, Ann. Eugen. 7, 355 (1937).

[5] I. Sendiña-Nadal, M. Gómez-Gesteira, V. Pérez-Muñuzuri, V. Pérez-Villar, J. Armero, L. Ramírez-Piscina, J. Casademunt, F. Sagués, and J.M. Sancho, Phys. Rev. E 56, 6298 (1997).

[6] O. Steinbock, V.S. Zykov, and S.C. Müller, Phys. Rev. E 48, 3295 (1993).

[7] I. Schebesch and H. Engel, Phys. Rev. E 57, 3905 (1998).

[8] J. Xin, SIAM Rev. 42, 161 (2000).

[9] N. Shigesada, K. Kawasaki, and E. Teramoto, J. Theor. Biol. 79, 83 (1979); Theor Popul. Biol. 30, 143 (1986); N. Shigesada and K. Kawasaki, Biological Invasions: Theory and Practice (Oxford University Press, Oxford, 1986).

[10] K.R. Swanson, E.C. Alvord, Jr., and J.D. Murray, Acta Biotheor. 50, 223 (2002); Cell Prolif 33, 317 (2000).
[11] L. Edelstein-Keshet and A. Spiros, J. Theor. Biol. 216, 301 (2002).

[12] K.I. Nakamura, H. Matano, D. Hilhorst, and R. Schätzle, J. Stat. Phys. 95, 1165 (1999); J. Norbury and L.C. Yeh, Nonlinearity 14, 849 (2001); SIAM (Soc. Ind. Appl. Math.) J. Appl. Math. 61, 1418 (2001).

[13] J.P. Keener, Physica D 136, 1 (2000).

[14] J.P. Keener, SIAM (Soc. Ind. Appl. Math.) J. Appl. Math. 61, 317 (2000).

[15] I. Mitkov, K. Kladko, and J.E. Pearson, Phys. Rev. Lett. 81, 5453 (1998).

[16] H.G. Rotstein, A.M. Zhabotinsky, and I.R. Epstein, Chaos 11, 833 (2001).

[17] S. Ponce Dawson, J. Keizer, and J.E. Pearson, Proc. Natl. Acad. Sci. U.S.A. 96, 6060 (1999).

[18] S.V. Petrovskii, Tech. Phys. Lett. 19, 397 (1993).

[19] A. Lemarchand, A. Lesne, and M. Mareschal, Phys. Rev. E 51, 4457 (1995); J. Armero, J.M. Sancho, J. Casademunt, A.M. Lacasta, L. Ramírez-Piscina, and F. Sagués, Phys. Rev. Lett. 76, 3045 (1996); A. Rocco, J. Casademunt, U. Ebert, and W. van Saarloos, Phys. Rev. E 65, 012102 (2002). 
[20] W. van Saarloos, Phys. Rev. A 37, 211 (1988).

[21] R.D. Benguria and M.C. Depassier, Phys. Rev. Lett. 73, 2272 (1994).

[22] Consider the problem $\partial_{t} \phi=D \partial_{x x} \phi+U g(x / l) f(\phi)$ where $D$ is the diffusion coefficient (constant), $U$ the reaction rate (constant), $g(x)$ the heterogeneity function, and $l$ the characteristic length of the spatial heterogeneities. If we define the dimensionless variables $x^{*}=x \sqrt{U / D}$ and $t^{*}=t U$ then the above equation, omitting asterisks for notational simplicity, takes the form $\partial_{t} \phi=\partial_{x x} \phi+g(\varepsilon x) f(\phi)$ where $\varepsilon \equiv l^{-1} \sqrt{D / U}$. The width of the front $L \sim\left(\partial_{x} \phi\right)^{-1}=\sqrt{U / D}\left(\partial_{x} * \phi\right)$ is $O(\sqrt{U / D})$, then $\varepsilon$ is $O(L / l)$. So that, when $L \ll l$ then $\varepsilon \ll 1$.

[23] U. Ebert and W. van Saarloos, Phys. Rev. Lett. 80, 1650 (1998); Physica D 146, 1 (2000); Phys. Rep. 337, 139 (2000).

[24] L.C. Evans, Partial Differential Equations (American Mathematical Society, Providence, 1998).
[25] V. Méndez and J.E. Llebot, Phys. Rev. E 56, 6557 (1997).

[26] V. Méndez, J. Fort, and J. Farjas, Phys. Rev. E 60, 5231 (1999).

[27] K.M. Vereecken, E.J. Dens, and J.F. Van Impe, J. Theor. Biol. 205, 53 (2000).

[28] M. Freidlin, Functional Integration and Partial Differential Equations (Princeton University Press, Princeton, NJ, 1985).

[29] J. Gärtner, Math. Nachr. 112, 125 (1983).

[30] L.C. Evans and P.E. Souganidis, Indiana Univ. Math. J. 33, 773 (1984); 38, 141 (1989); Ann. Inst. Henri Poincaré, Sect. A 6, 229 (1989).

[31] J. Fort and V. Méndez, Phys. Rev. Lett. 82, 867 (1999); Phys. Rev. E 60, 5894 (1999).

[32] O’Shaughnessy and I. Procaccia, Phys. Rev. Lett. 54, 455 (1985); Phys. Rev. A 32, 3073 (1985) 